\title{
Lower mutant-allele tumor heterogeneity is a biomarker in FGFR3-mutant bladder cancer for better prognosis
}

\author{
Yuying Han ${ }^{1}$, Xu Liu', Haihong Ye ${ }^{1 *}$, Ye Tian ${ }^{2^{*}}$ and Zhengguo $\mathrm{Ji}^{2^{*}}$
}

\begin{abstract}
Background: Bladder cancer displays a broad mutational spectrum and intratumor heterogeneity (ITH), which results in difference in molecular phenotypes and resistance to therapies. However, there are currently no clinically available measures to predict patient prognosis using ITH. We aimed to establish a clinically relevant biomarker by using ITH for informing predictive of outcomes.
\end{abstract}

Methods: We used the Bioconductor R package Maftools to efficiently and comprehensively analyze somatic variants of muscle-invasive bladder cancer (MIBC) from The Cancer Genome Atlas (TCGA). We then used a mutantallele tumor heterogeneity (MATH) algorithm to measure ITH and explored its correlation with clinical parameters as well as mutational subtypes.

Results: We observed a broad range of somatic mutations in MIBC from TCGA. MATH value was higher for the high-grade group than for the low-grade group $(p<0.05)$. There was a strong correlation between higher MATH value and presence of TP53 mutations $(p=0.008)$, as well as between lower MATH value and presence of FGFR3 mutations $(p=0.006)$. Patients with FGFR3 mutation and low MATH value exhibit longer overall survival time than that of all BLCA patients $(p=0.044)$, which was replicated in another bladder cancer database composed of 109 BLCA patients.

Conclusion: Measures of tumor heterogeneity may be useful biomarkers for identifying patients with bladder cancer. Low MATH value was an independent risk factor that predicted better prognosis for patients with FGFR3 mutation compared to all BLCA patients.

Keywords: Mutant-allele tumor heterogeneity, FGFR3, Prognosis, Bladder cancer

\footnotetext{
*Correspondence: yehaihong@ccmu.edu.cn; tianye@ccmu.edu.cn; jizhengguo@ccmu.edu.cn

${ }^{1}$ Department of Medical Genetics and Developmental Biology, School of Basic Medical Sciences, Beijing Key Laboratory of Neural Regeneration and Repair, Capital Medical University, Beijing, China ²Department of Urology, Beijing Friendship Hospital, Capital Medical University, Beijing, China
}

(c) The Author(s). 2020 Open Access This article is licensed under a Creative Commons Attribution 4.0 International License, which permits use, sharing, adaptation, distribution and reproduction in any medium or format, as long as you give appropriate credit to the original author(s) and the source, provide a link to the Creative Commons licence, and indicate if changes were made. The images or other third party material in this article are included in the article's Creative Commons licence, unless indicated otherwise in a credit line to the material. If material is not included in the article's Creative Commons licence and your intended use is not permitted by statutory regulation or exceeds the permitted use, you will need to obtain permission directly from the copyright holder. To view a copy of this licence, visit http://creativecommons.org/licenses/by/4.0/. The Creative Commons Public Domain Dedication waiver (http://creativecommons.org/publicdomain/zero/1.0/) applies to the data made available in this article, unless otherwise stated in a credit line to the data. 


\section{Introduction}

Bladder cancer is one of the most common and highly intratumor heterogeneous malignant tumors of the genitourinary system. Bladder urothelial carcinoma is the most common type, accounting for greater than $90 \%$ of all bladder cancers [1]. The majority of bladder cancers occur in men, and there is a wide variation in the incidence and mortality rates worldwide [2]. Based on the degree of tumor invasion, bladder cancers can be categorized as non-muscle-invasive bladder cancer (NMIBC) and muscle-invasive bladder cancer (MIBC). Both NMIBC and MIBC are major sources of morbidity and mortality worldwide. MIBC is associated with greater malignancy, a more diverse mutational spectrum, higher recurrence rate, and worse overall prognosis. Molecular markers have shown potential value in improving diagnostic accuracy of risk stratification of patients $[3,4]$.

Intratumor heterogeneity (ITH) plays a pivotal role in driving progression and therapeutic resistance in bladder cancer [5]. ITH produces different molecular phenotypes, presenting a significant challenge in the implementation of precision medicine which holds the promise to transform oncology through the use of molecular markers to inform prognosis and guide treatment [6-8].

Based on mutation and gene expression signatures, MIBC can be grouped into five mRNA molecular expression subtypes: luminal-papillary, luminal-infiltrated, luminal, basal-squamous, and neuronal $[9,10]$. Molecular subtyping may predict patient survival [9] and response to conventional neoadjuvant chemotherapy [11] and is thus potentially clinically important. FGFR3 and TP53 mutations are the most common mutations in MIBC but are mutually exclusive events. Usually, FGFR3 mutations are accompanied by fewer molecular alterations than are found in FGFR3 wild-type tumors [9, 12, 13]. FGFR3-mutant tumors are significantly correlated with lower-grade bladder cancer, while TP53-mutant tumors were found to be strongly associated with a later tumor stage and higher tumor grade. Interestingly, FGFR3 mutations have been predominantly found in genetically stable bladder cancers with favorable prognoses [12]. Furthermore, anti-FGFR3 therapy slows bladder cancer growth, especially in FGFR3-mutant tumors [14]. FGFR3 represents a potentially significant actionable therapeutic target [15], and FGFR3 can distinguish MIBC subgroup and response to neoadjuvant chemotherapy [16] . However, the heterogeneity of FGFR3 genotypes within a tumor has not been adequately addressed and may negatively impact therapeutic response [17].

In the present study, we downloaded somatic variants of MIBC in Mutation Annotation Format from the Cancer Genome Atlas (TCGA), and used Maftools
[18] to efficiently and comprehensively analyze somatic variants in bladder cancer. We then used a mutant-allele tumor heterogeneity (MATH) algorithm [19] to measure ITH and explore its correlation with clinical parameters. Our integrative analysis reveals the clinical and genetic relevance of ITH in bladder cancer. We identify low MATH value as an independent risk factor that predicts the outcome of FGFR3mutant subtype MIBC patients, which was verified in an independent MIBC database.

\section{Materials and methods \\ Patients and clinical variables}

The publicly available mutation data and clinical data used in this study were released by TCGA Data Portal (https: //portal.gdc.cancer.gov) and downloaded from cBioPortal at [http://www.cbioportal.org/datasets]. Another cohort of mutation and clinical data for validation was downloaded from cBioPortal at [http://www.cbioportal.org/datasets].

\section{Genomic variants and MATH calculation}

We downloaded MIBC somatic variants in Mutation Annotation Format (MAF) from the Cancer Genome Atlas (TCGA). We used Maftools, an R Bioconductor package that can be used for integrative analysis of somatic variants, to efficiently and comprehensively analyze somatic variants in bladder cancer.

We calculated the MATH value for each bladder cancer sample according to a previously described protocol $[19,20]$. The steps used to determine the MATH value can be summarized as follows: (1) calculate the mutantallele fraction (MAF) for each locus as the ratio of mutant reads to total reads; (2) obtain the absolute difference of each MAF from the median MAF value and multiply the median of these absolute differences by a constant factor (1.4826) to generate the median absolute deviation (MAD); (3) calculate the MATH value as the ratio of the MAD to the median of the MAFs of the tumor's mutated genomic loci, presented as a percentage $(\mathrm{MATH}=100 * \mathrm{MAD} /$ median$)$.

\section{Statistical analysis}

Wilcoxon rank-sum test was used to analyze differences among different variant types. The associations between MATH value and clinical characteristics were evaluated using a Kruskal-Wallis test. Kaplan-Meier survival analyses and log-rank tests were used to estimate the prognoses. All other statistical analyses were performed using IBM SPSS Statistics 25 software. $p$ values less than 0.05 were considered statistically significant. 


\section{Results}

Clinical characteristics of patients with bladder cancer We analyzed genome sequencing data from 412 patients with bladder cancer obtained from the TCGA Bladder Cancer (BLCA) Data Portal. The clinical and pathological characteristics of all patients included in the current analysis are shown in Table 1. High-grade tumors comprised $94.9 \%$ of the analysis cohort, whereas low-grade tumors comprised the remaining $5.1 \%$.

\section{Comprehensive analysis of somatic variants in bladder} cancer

A total of 87,624 somatic mutations were identified using Maftools. These variants were comprised of 75,116 missense mutations, 7291 nonsense mutations, 1216 insertions, 1,982 deletions, 130 translation start site variants, 1762 splice site variants, and 127 nonstop mutations (Fig. 1a, b, and d). These mutations were further classified using the variant effect predictor (Fig. 1c). The median of total mutation number obtained from each sample was 148 (Fig. 1a, e). There were significant differences among different variant types (Fig. 1f). We identified 50 genes that were mutated in $>10 \%$ of samples using Maftools: TP53 (47\%), TTN (45\%), MUC16 (28\%), KDM6A (26\%), SYNE1 (20\%), RB1 (18\%), FGFR3 (14\%), STAG2 (14\%), BIRC6 (11\%), RYR1 (10\%), ADGRV1 (10\%), and AHNAK (10\%) (Fig. 1b and Table S1).

\section{MATH value characteristics and their relationship with clinical factors}

Analysis of the mutation rates of various tumors in TCGA database revealed a high mutation rate for

Table 1 Correlation analysis between clinical characteristics and MATH values in the TCGA-BLCA project

\begin{tabular}{|c|c|c|c|c|}
\hline Characteristics & Number of patients & Percent (\%) & $\mathrm{MATH} \pm \mathrm{SD}$ & $p$ value \\
\hline Age, median (IQR) & $408(34-90)$ & & & $p=0.388$ \\
\hline$\leq 50$ & 25 & 6.1 & $41.5 \pm 19$ & \\
\hline $51-74$ & 254 & 61.3 & $46.1 \pm 16$ & \\
\hline$\geq 75$ & 133 & 32.6 & $46.5 \pm 13$ & \\
\hline Gender & & & & $p=0.527$ \\
\hline Male & 304 & 73.8 & $46.2 \pm 16$ & \\
\hline Female & 108 & 26.2 & $45.2 \pm 13$ & \\
\hline Race & & & & $p=0.484$ \\
\hline Asian & 44 & 11.2 & $43.3 \pm 19$ & \\
\hline Caucasian & 327 & 83.0 & $46.0 \pm 14$ & \\
\hline African & 23 & 5.8 & $45.6 \pm 16$ & \\
\hline Unkown & 18 & Unkown & Unkown & \\
\hline Histologic subtype & & & & $p=0.162$ \\
\hline Papillary & 134 & 32.8 & $44.4 \pm 18$ & \\
\hline Non-papillary & 274 & 67.2 & $46.7 \pm 14$ & \\
\hline Unkown & 5 & Unkown & Unkown & \\
\hline Stage & & & & $p=0.425$ \\
\hline Stage I & 2 & 0.5 & $49.5 \pm 50$ & \\
\hline Stage II & 131 & 32.0 & $44.3 \pm 16$ & \\
\hline Stage III & 141 & 34.4 & $45.6 \pm 15$ & \\
\hline Stage IV & 136 & 33.2 & $47.4 \pm 13$ & \\
\hline Unkown & 2 & Unkown & Unkown & \\
\hline Grade & & & & $p=0.024$ \\
\hline High-grade & 388 & 94.9 & $46.2 \pm 15$ & \\
\hline Low-grade & 21 & 5.1 & $38.6 \pm 18$ & \\
\hline Unkown & 3 & 1 & & \\
\hline Recurrence & & & & $p=0.389$ \\
\hline Recur & 147 & 45.1 & $46.8 \pm 15$ & \\
\hline Free & 179 & 54.9 & $45.3 \pm 16$ & \\
\hline Unkown & 86 & Unkown & Unkown & \\
\hline
\end{tabular}




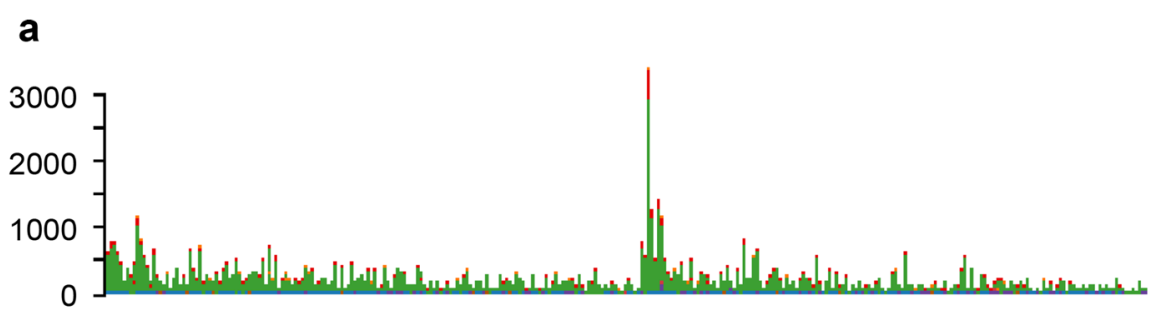

b

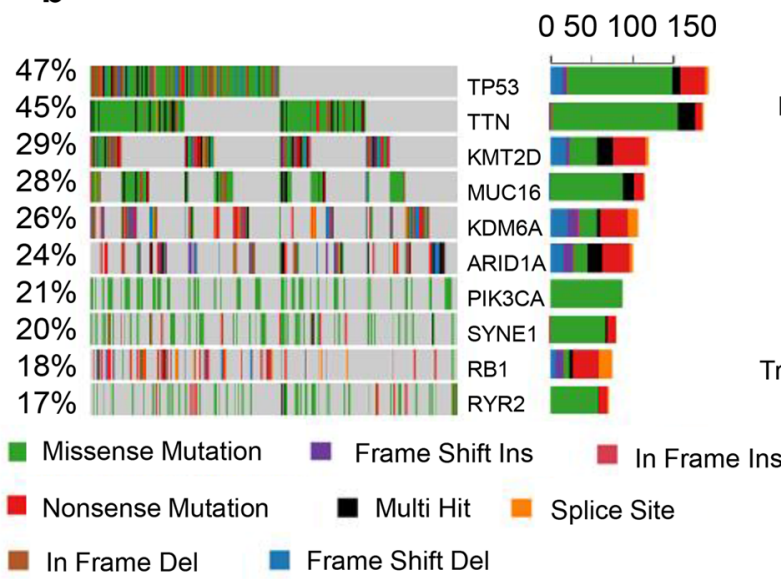

d

Variant Type

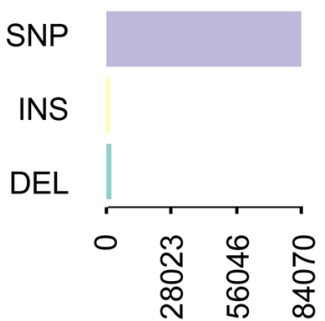

e

Variants per Sample Median: 148

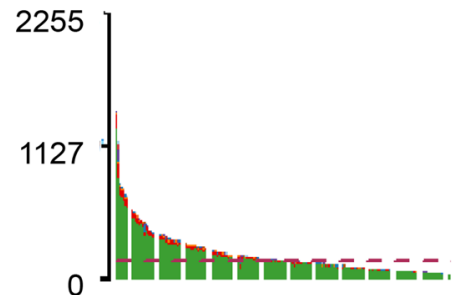

C

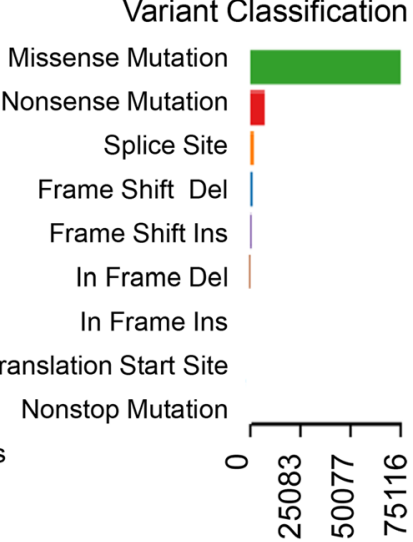

f

Variant Classification
Summary

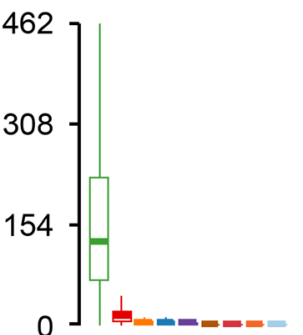

Fig. 1 Characteristics of variants identified in the BLCA study cohort. a Cumulative frequencies of variants in individual BLCA cases. b Oncoplot displaying the somatic landscape of the BLCA cohort. Genes are ordered by their mutation frequency. The side bar plot displays $Q$ values estimated by MutSigCV. c Bundled bar chart classifying variant types using the variant effect predictor (VEP). d Stacked bar chart summarizing the variant types of all cases with substitutions, insertions, and deletions. e Histogram showing the cumulative frequency of variants for individual cases. The median of mutation number per sample is 148. $\mathbf{f}$ Statistical analysis of differences among different variant types (Wilcoxon rank-sum test, $p<0.0001)$

bladder cancer. The median mutational burden for the bladder cancer samples was 148 mutations, which is significantly greater than those of many other cancer types listed in TCGA dataset, ranking fourth in the TCGA tumor category (Fig. 2a). Bladder cancer also displays high ITH during its progression. ITH can be assessed using MATH value. MATH values are calculated from the median absolute deviation (MAD) and the median of its mutant-allele fractions of tumor-specific mutated loci, so the precision of MATH values depends on the sampling of loci and of mutant vs. reference alleles [19]. Thus, MATH value represents a different aspect of tumor biology than the mutation rate. MATH value has been shown to be a simple, quantitative, and generally applicable approach to evaluate the degree of ITH. Here, we calculated MATH values in the TCGA bladder cancer cohort to evaluate the clinical implication of ITH in bladder cancer. The distribution of the MATH values is shown in Fig. 2b. A Q-Q (quantile-quantile) plot (Fig. $2 c)$ and Kolmogorov-Smirnov test $(p=0.2)$ both show 

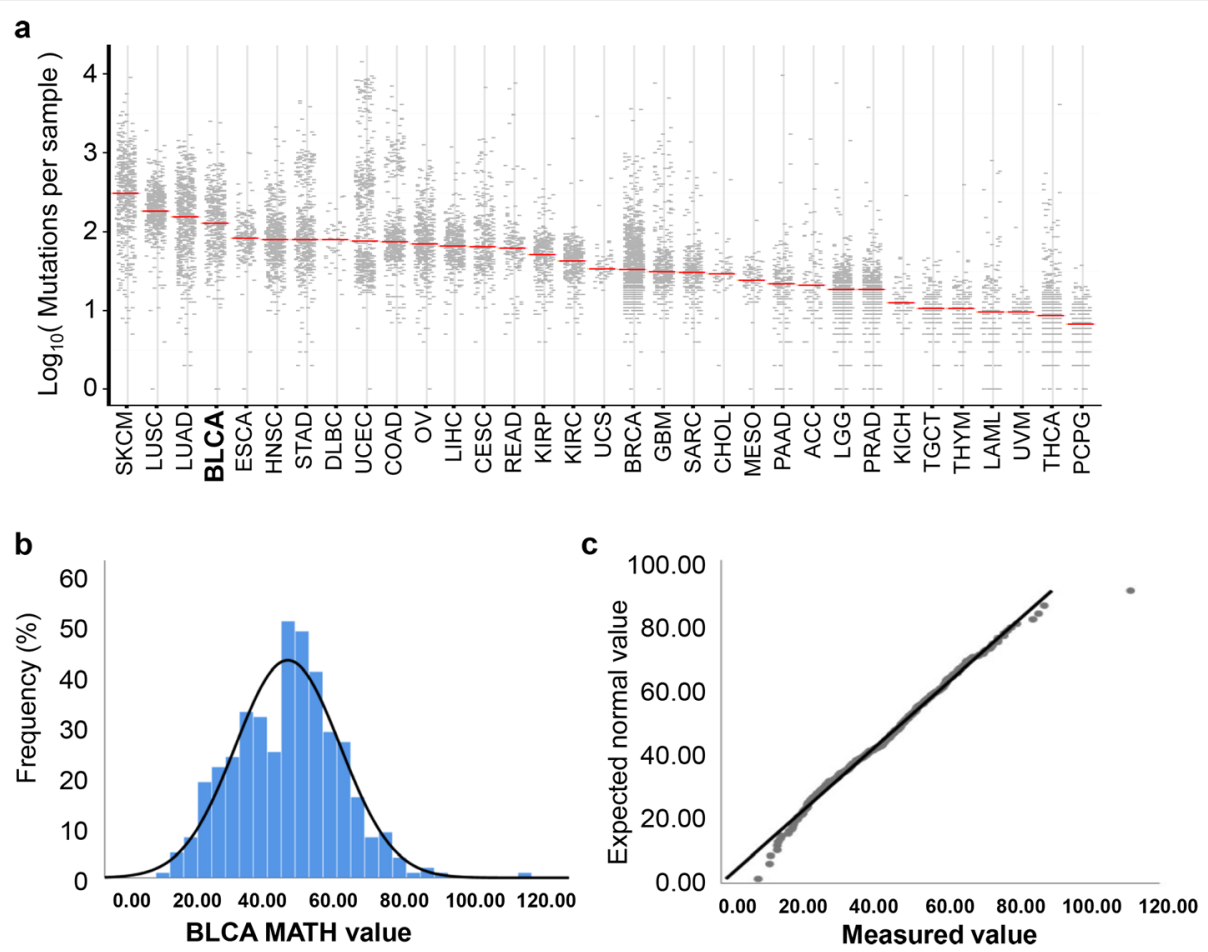

Fig. 2 Characteristics of the variants in the BLCA study cohort. a Scatter plot comparing the mutational loads of the BLCA cohort with other TCGA cancer cohorts. b Distribution of the mutant-allele tumor heterogeneity (MATH) values among bladder cancer patients. c Q-Q (quantilequantile) plot analysis of the distribution of all MATH values

that the MATH values from this cohort fit a normal distribution.

The upper and lower tertiles of the MATH values were 39.4 and 52.3 , respectively. Thus, cases with MATH values less than 39.4 were classified into the "low MATH" group (135 patients; 33.3\%) and cases with a MATH value greater than 52.3 were classified into the "high MATH" group (135 patients; 33.3\%), while the remainder (MATH value between 39.4 and 52.3) were defined as the "intermediate MATH" group (136 patients; 33.4\%). First, we examined the correlation between
MATH value and overall survival time in each patient using linear regression and found that MATH value did not exhibit a linear dependence relation with patient's overall survival time ( $p=0.646$, Fig. 3a). Next, we investigated the prognostic significance of MATH value in the three predefined MATH groups described above. We find that MATH value was not an independent predictor of overall survival time in the entire bladder cancer cohort ( $p=0.725$, Fig. $3 \mathrm{~b})$.

Because the MATH value grouped by grade, stage, or race did not conform to normal distribution, rank sum
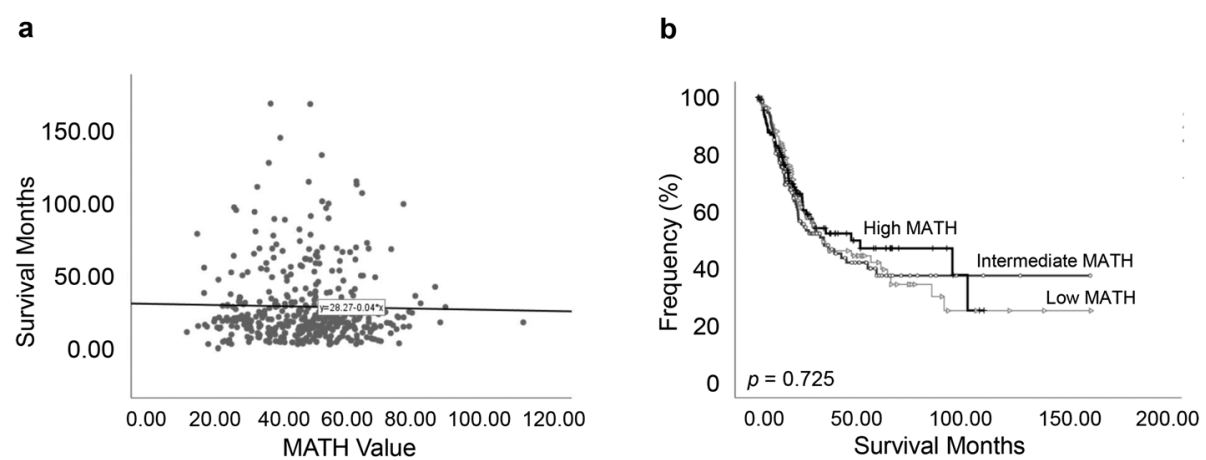

Fig. 3 Mutant-allele tumor heterogeneity (MATH) value and its relationship with survival time. a Linear dependence relation between MATH value and survival time $(p=0.646)$. $\mathbf{b}$ Kaplan-Meier survival curves for groups with low, intermediate, and high MATH values. Groups are based on the MATH value cutoffs of the upper and lower tertiles of all MATH values. $p=0.725$ 
test was used to test the difference among them. Using rank sum test of variance, the MATH value was found to be significantly related to tumor grade $(p=0.024)$, but not to race $(p=0.484)$ or stage $(p=0.425)$ (Table $1)$. Using one-way ANOVA, the MATH value was not found to be related to recurrence $(p=0.389)$ or tumor histologic subtype ( $p=0.162)$ (Table 1$)$. Figure 4a shows the range of calculated MATH values for tumors initially labeled low-grade and high-grade. Higher MATH values were specifically related to a high tumor grade. MATH value was higher in the high-grade group than in the low-grade group $(p<0.05)$, which suggests that MATH value may be useful in differentiating such patients. We next analyzed the percentage of low- and high-grade cases with different ranges of tumor MATH values. The high-grade cases tended to show a significantly increased level of MATH value than that of the low-grade cases (Fig. 4b). We then explored the correlation of MATH value and survival in low-grade and high grade subgroups. Interestingly, MATH value was not an independent prognostic factor in the low- and high-grade patients (Fig. 4c, $p=0.116$ ). Taken together, we found that MATH value was not an independent risk factor for survival time, but a high MATH value was related to high-grade BLCA.

\section{Low MATH value was an independent favorable} prognostic biomarker in FGFR3-mutant patients

Although MATH value was not related to the overall mutation rate, we hypothesized that different driver gene mutations may direct different routes of tumor evolution, resulting in variable intratumor heterogeneity. To test this, we analyzed the mutual exclusivity and cooccurrence of the top 50 mutated genes in BLCA based on cBioPortal $[21,22]$ (Table 2). We observed mutually exclusive variants in FGFR3 vs. TP53 $(q<0.003)$ and FGFR3 vs. RB1 $(q<0.02)$. Similar analyses identified cooccurrence of variants in TP53 vs. RB1, RYR1 vs. AHNAK, BIRC6 vs. ADGRV1, SYNE1 vs. AHNAK, FGFR3 vs. STAG2, KDM6A vs. STAG2, MUC16 vs. BIRC6, and TTN vs. MUC16 $(q<0.005)$. In the BLCA cohort, TP53 was mutated in $>47 \%$ of the samples, and FGFR3 was mutated in $>14 \%$ of the samples. FGFR3 and TP53 mutation are potential survival prediction biomarkers of MIBC. The known role of the p53, as a guardian of the genome stability [23], supports the general hypothesis that TP53 mutations lead to increased ITH. Thus, we first aimed to validate the hypothesis that TP53 mutations, rather than FGFR3 mutations, was associated with greater ITH in BLCA. Based on the presence of somatic mutations in TP53 and FGFR3, we divided the BLCA cohort into three groups: TP53-mutant, FGFR3-mutant, and no TP53 and FGFR3-mutant. Using MATH value as a measure of ITH, we examined the relationship of heterogeneity between the above three groups. Consistent with our hypothesis, TP53 mutations were specifically associated with higher MATH values. MATH values were higher in the BLCA cases with TP53 mutations than in those with FGFR3-mutant (Fig. $5 \mathrm{a}, p<0.001$ ) or no TP53- and FGFR3-mutant (Fig. $5 \mathrm{a}, p<0.001)$ group. MATH values were lowest in BLCA cases with FGFR3 mutations (Fig. 5a).

Next, we investigated the prognostic significance of MATH value in the TP53-mutant and the FGFR3-mutant groups. For FGFR3-mutant patients, we observed a trend of increased survival time compared to all patients in the BLCA cohort, although the change was not significant (Fig. 5b, $p=0.149$ ). We then divided the TP53and FGFR3-mutant groups into low and high MATH value subgroups, respectively, using quartile MATH
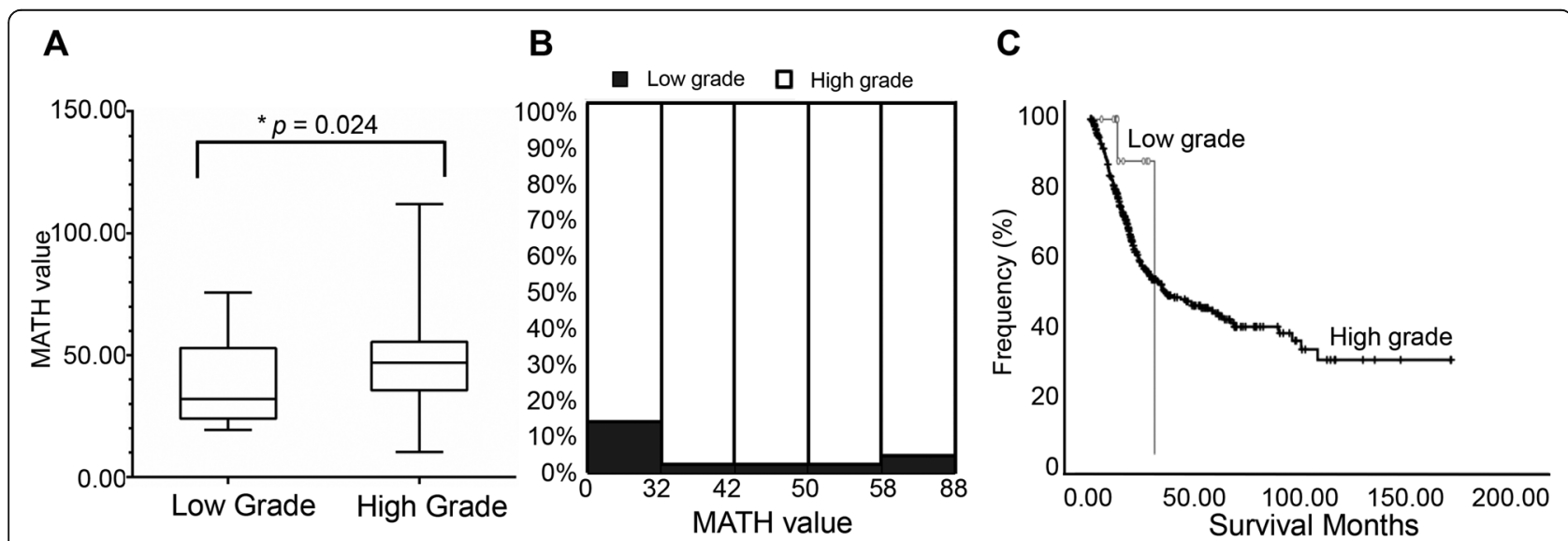

Fig. 4 Mutant-allele tumor heterogeneity (MATH) value and its relationship with clinical grade. a MATH values for the original low-grade and high-grade tumors $(p=0.024)$. b MATH value and its relationship with clinical grade. Each panel represents the relationship between clinical grade and MATH values. Within each panel, the horizontal axis represents the range of tumor MATH values divided into five groups comprised of approximately equal numbers of tumors. c Kaplan-Meier survival curves for the low-grade and high-grade groups ( $p=0.116)$ 
Table 2 Mutual exclusivity and co-occurrence of the top 50 mutated genes in BLCA

\begin{tabular}{llllllllll}
\hline A & B & Neither & A not B & B not A & Both & Log $_{\mathbf{2}}$ odds ratio & $\boldsymbol{p}$ value & $\boldsymbol{q}$ value & Tendency \\
\hline TP53 & FGFR3 & 169 & 185 & 45 & 13 & -1.922 & $<0.001$ & 0.003 & Mutual exclusivity \\
RB1 & FGFR3 & 284 & 70 & 56 & 2 & -2.787 & $<0.001$ & 0.02 & Mutual exclusivity \\
TP53 & RB1 & 199 & 141 & 15 & 57 & 2.423 & $<0.001$ & $<0.001$ & Co-occurrence \\
RYR1 & AHNAK & 337 & 33 & 25 & 17 & 2.796 & $<0.001$ & $<0.001$ & Co-occurrence \\
BIRC6 & ADGRV1 & 333 & 34 & 28 & 17 & 2.572 & $<0.001$ & 0.001 & Co-occurrence \\
SYNE1 & AHNAK & 307 & 63 & 21 & 21 & 2.285 & $<0.001$ & 0.002 & Co-occurrence \\
FGFR3 & STAG2 & 317 & 38 & 37 & 20 & 2.173 & $<0.001$ & 0.002 & Co-occurrence \\
KDM6A & STAG2 & 277 & 78 & 28 & 29 & 1.879 & $<0.001$ & 0.002 & Co-occurrence \\
MUC16 & BIRC6 & 272 & 89 & 23 & 28 & 1.896 & $<0.001$ & 0.003 & Co-occurrence \\
TTN & MUC16 & 174 & 121 & 43 & 74 & 1.307 & $<0.001$ & 0.005 & Co-occurrence \\
\hline
\end{tabular}

values as cut-offs. We found that low MATH value was a significant predictor of better survival in the FGFR3mutant group (Fig. $5 c, p<0.05$ ). On the contrary, we found no significant difference of overall survival in FGFR3-mutant with high MATH value compared to all patients in the BLCA cohort (Fig. $5 \mathrm{~d}, p=0.925$ ), which indicated that the better survival of patients was specifically correlated with patients carrying FGFR3 mutation with lower MATH value. At the same time, we compared the overall survival between TP53-mutant and all patients in the BLCA cohort. There was no significant difference between them (Fig. 5e, $p=0.761$ ). Moreover, there was no significant difference in overall survival comparing TP53-mutant with either low MATH value or high value to all patients in the BLCA cohort, respectively (Fig. 5f, $p=0.639$; Fig. 5g, $p=0.626$;), which indicated that MATH value was not a biomarker for prognosis in TP53-mutant patients. Taken together, we found that low MATH value was an independent favorable prognostic biomarker in FGFR3-mutant patients.

Validation of low MATH value as a prognostic biomarker in FGFR3-mutant patients in an independent BLCA cohort To further investigate whether low MATH value in FGFR3-mutant patients is widely applicable to predict better overall survival in BLCA, we selected another BLCA cohort which was composed of 109 bladder cancer patients published in 2015 [24]. We download all mutation and clinical data from cBioPortal and reassessed its prognostic value. First, to verify that FGFR3 mutations, rather than TP53 mutations, were associated with lower ITH in BLCA, we
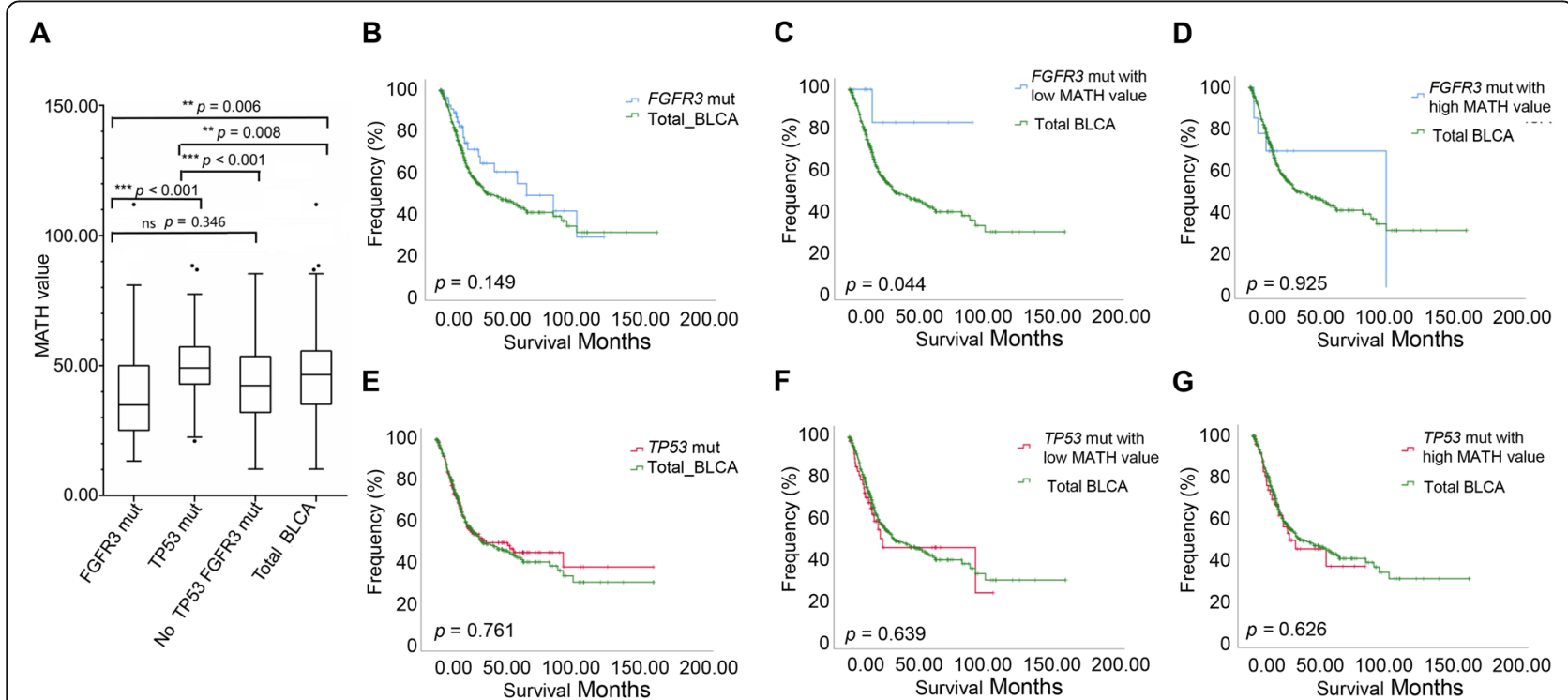

Fig. 5 Prognostic evaluation of MATH value in TP53 and FGFR3 mutations patients. a MATH values for TP53-mutant, FGFR3-mutant, and no mutation with TP53 and FGFR3, Kruskal-Wallis test $(p<0.05)$. b-g Kaplan-Meier survival analysis comparing patients in specific subgroups to all patients in the BLCA cohort from TCGA 
divided the 109 samples [24] into three groups: TP53-mutant, FGFR3-mutant, and no TP53 and FGFR3-mutant. Using MATH as a measure of ITH, we examined the relationship of heterogeneity between the above three groups. Although there was no significant difference between them $(p>0.05)$, probably due to the small sample size in this cohort, the overall trend of the median MATH values showed that MATH values were greater in BLCA patients with TP53 mutations than in those with FGFR3 mutations (Fig. 6a, $p=0.074$ ) and MATH values were lowest in BLCA cases with FGFR3 mutations (Fig. 6a), consistent with our findings in the TCGA BLCA cohort (Fig. 5a).

Next, we investigated the prognostic significance of MATH value in the FGFR3-mutant and TP53-mutant groups. In all FGFR3-mutant patients, we observed a trend of increased survival time compared to all patients in the whole cohort (Fig. 6,b $p=0.279$ ). We divided FGFR3-mutant patients and TP53-mutant patients into low MATH value subgroup and high MATH value subgroup, respectively. The prognosis trend of FGFR3-mutant patients with low MATH value was better than that of the patients in the whole cohort, although the difference was not statistically significant, probably due to the limited number of patients in this subgroup (Fig. 6c, $p=$ 0.170 ). We found no significant difference of overall survival in any other subgroups (Fig. 6e-g), indicating that MATH value was not a biomarker for prognosis in the TP53-mutant patients. Taken together, we verified that low MATH value was an independent favorable prognostic biomarker in FGFR3-mutant patients in another bladder cancer cohort.

\section{Discussion}

Bladder cancer, including both NMIBC and MIBC, is a major source of morbidity and mortality worldwide. MIBCs have a more diverse mutation spectrum $[25,26]$ and display high overall mutation rates similar to those of non-small cell lung cancers and melanoma [27]. MIBCs are also highly recurrent cancers worldwide [28].

Similar to most solid tumors, BLCA is spatially heterogeneous. ITH is the result of temporal acquisition of mutations and corresponding tumor evolution. ITH is observed at both the genomic and the transcriptomic levels. ITH plays a pivotal role in driving bladder cancer progression and resistance to therapies. High genetic heterogeneity is thought to contribute to risk of poor survival in patients with bladder cancers. MATH value is a novel approach to quantify ITH. In several previous studies, it was reported as a prognostic biomarker for patients with head and neck squamous cell carcinoma $[19,20]$. Any personalized prognostic indicator or tumor treatment requires a test to distinguish patients who will benefit from those who will not [29]. However, differences in ITH in MIBC evaluated using MATH value have not been thoroughly explored at the genomic level. Our study represents the first attempt to describe the prognostic value of MATH value.

Precision medicine is transforming oncology through the use of molecular markers to guide treatment and inform prognosis [6]. MIBC has a more diverse mutant

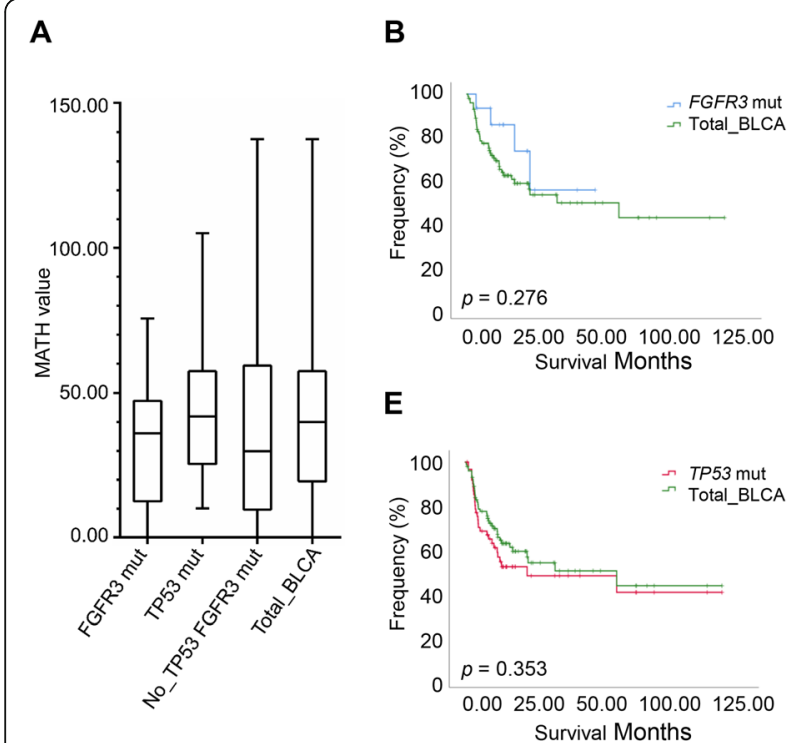

C

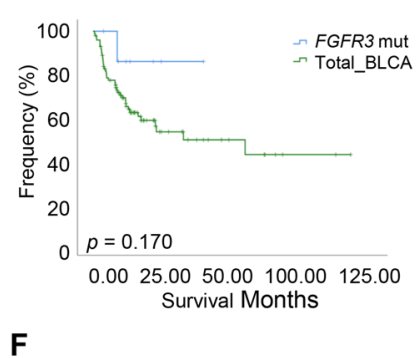

$\mathbf{F}$

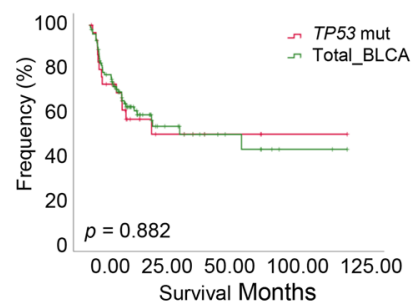

D

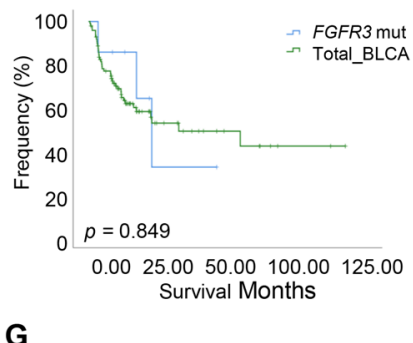

G

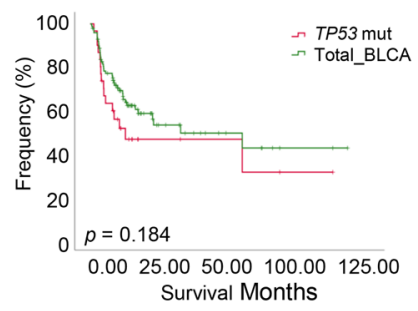

Fig. 6 Validation and prognostic evaluation of MATH value in TP53 and FGFR3 mutation patients in an independent BLCA cohort. a MATH values for TP53-mutant, FGFR3-mutant, and no mutation with TP53 and FGFR3, Kruskal-Wallis test $(p>0.05)$. b-g Kaplan-Meier survival analysis comparing patients in specific subgroups to all patients in an independent BLCA cohort 
spectrum (as shown in Table S1). Different driver gene mutations may direct different routes of tumor evolution which may affect the prognosis of bladder cancer patients. MATH value was not related to the overall mutation rate and depended on the mutation frequency of multiple mutated genes. Therefore, the MATH value was not the only factor affecting the prognosis of bladder cancer patients, and the mutation of the driver gene needs to be considered. FGFR3 and TP53 are the most common mutations in MIBC, but are mutually exclusive events. Therefore, we combined FGFR3 and TP53 mutations and MATH value to analyze the prognosis of bladder cancer patients. FGFR3 mutation is usually accompanied by fewer molecular alterations than wildtype FGFR3 [12, 13]. The p53 protein functions as a guardian of the genome stability, which supports the hypothesis that mutations in TP53 result in increased ITH. We examined the relationship between heterogeneity (using MATH value) with FGFR3 and/or TP53 mutations and found that FGFR3 mutations were associated with lower ITH. The administration of neoadjuvant chemotherapy preceding radical cystectomy improves overall survival of patients with MIBC [30], and FGFR3 mutations in MIBCs are potential predictive biomarkers of the response to neoadjuvant [16]. In most primary invasive bladder cancers, FGFR3 status can guide the selection of FGFR targeted therapy [31]. Our study showed that FGFR3-mutatant cases have the characteristics of low MATH value, which provides a valuable reference standard for neoadjuvant chemotherapy. We also assessed the prognostic value of MATH with TP53 or FGFR3 mutations and found that a low MATH value was an independent risk factor that predicted better survival in the FGFR3-mutant patients. This may be due to better sensitivity to cisplatin and gemcitabine treatment in this subgroup of patients, which requires further clinical and genetic studies to verify.

Given the nature of available BLCA cohorts, several potential limitations exist in our current analysis. Although properly powered, the bladder cancer cohorts used in our study lacked several clinic pathological parameters. The follow-up data of patients are not uniform, so there are some restrictions on the clinical correlation analysis. Moreover, the information on the pre- and postoperative treatment is not available in the majority of the patients with bladder cancer, and thus, these impacts on overall survival analysis could not be fully considered. Furthermore, the majority of BLCA patients were of the Caucasian race, so the predictive significance for other populations is questionable. Further studies using clinical samples from other racial groups with complete clinical data and follow-up data are necessary, and further verification together with multiple algorithms are needed.
MATH value provides a quantifiable estimation of ITH and is less affected by the limitation of somatic mutation number and sequencing depth in the bladder cancer cohorts. However, the ITH represented by the MATH value might be confounded by the load of somatic copy number alterations, which should not be ignored. Using MATH value to quantify tumor heterogeneity is valuable, but the standards are different for each somatic caller in different databases, so the results from different databases are different. Therefore, the use of MATH value to quantify tumor heterogeneity must unify the adoption standard of somatic mutation.

In our study, we found that MATH value as a measure of ITH may be useful biomarkers for patients with bladder cancer. Here, we show that patients with FGFR3 mutations and low MATH value tend to exhibit favorable prognosis. ITH at the genome level should be taken into account to guide management of bladder cancer patients.

\section{Conclusions}

Bladder cancer is a malignant tumor with extensive mutant spectrum and high tumor heterogeneity. MATH value is effective in evaluating tumor heterogeneity. Measures of tumor heterogeneity may be useful biomarkers for identifying patients with bladder cancer. Low MATH value was an independent risk factor that predicted better prognosis for patients with FGFR3 mutation compared to all BLCA patients.

\section{Supplementary Information}

The online version contains supplementary material available at https://doi. org/10.1186/s12957-020-02084-3.

Additional file 1:Table S1 The top 50 mutated genes in BLCA.

\section{Abbreviations}

BLCA: Bladder cancer; ITH: Intratumor heterogeneity; MAF: Mutant-allele fraction; MATH: Mutant-allele tumor heterogeneity; MIBC: Muscle-invasive bladder cancer; NMIBC: Non-muscle-invasive bladder cancer; TCGA: The Cancer Genome Atlas

\section{Acknowledgments \\ The results of this study are based on data generated by The Cancer Genome Atlas (TCGA). We would like to thank "TCGA Research Network" for generating, curating, and providing the bladder cancer clinical data.}

\section{Authors' contributions}

$\mathrm{ZJ}, \mathrm{HY}$, and $\mathrm{YT}$ conceived the research, acquired the data, and drafted the manuscript. $\mathrm{YH}$ and $\mathrm{XL}$ performed the bioinformatics analysis and interpretation of the data. HY, ZJ, YT, and YH revised the paper for important content. All authors approved the final manuscript.

\section{Funding}

This work was supported by the Capital Health Research and Development of Special [2020-2-1111], National Natural Science Foundation of China [grant number 91854209; 31971116], and Beijing Municipal Natural Science

Foundation [grant number 5202005]. 


\section{Availability of data and materials}

The datasets used and/or analyzed during the current study are available from the corresponding author on reasonable request.

\section{Ethics approval and consent to participate}

Not applicable.

\section{Consent for publication}

Not applicable.

\section{Competing interests}

The authors declare that they have no conflict of interest.

Received: 18 August 2020 Accepted: 9 November 2020 Published online: 26 November 2020

\section{References}

1. Fleshner NE, Herr HW, Stewart AK, Murphy GP, Mettlin C, Menck HR. The National Cancer Data Base report on bladder carcinoma. The American College of Surgeons Commission on Cancer and the American Cancer Society. Cancer. 1996;78:1505-13.

2. Torre LA, Bray F, Siegel RL, Ferlay J, Lortet-Tieulent J, Jemal A. Global cancer statistics, 2012. CA Cancer J Clin. 2015;65:87-108.

3. Miremami J, Kyprianou N. The promise of novel molecular markers in bladder cancer. Int J Mol Sci. 2014;15:23897-908.

4. Zhu Y, Shi L, Chen P, Zhang Y, Zhu T. Identification of six candidate genes for endometrial carcinoma by bioinformatics analysis. World J Surg Oncol. 2020;18:161.

5. Warrick Jl, Sjodahl G, Kaag M, Raman JD, Merrill S, Shuman L, Chen G, Walter $V$, DeGraff DJ. Intratumoral heterogeneity of bladder cancer by molecular subtypes and histologic variants. Eur Urol. 2019;75:18-22.

6. Jackson SE, Chester JD. Personalised cancer medicine. Int J Cancer. 2015; 137:262-6.

7. Tsikrika FD, Avgeris M, Levis PK, Tokas T, Stravodimos K. Scorilas A: miR-221/ 222 cluster expression improves clinical stratification of non-muscle invasive bladder cancer (TaT1) patients' risk for short-term relapse and progression. Genes Chromosomes Cancer. 2018:57:150-61.

8. Pop-Bica C, Gulei D, Cojocneanu-Petric R, Braicu C, Petrut B, BerindanNeagoe I. Understanding the role of non-coding RNAs in bladder cancer: from dark matter to valuable therapeutic targets. Int J Mol Sci. 2017;18: 1514-38.

9. Robertson AG, Kim J, Al-Ahmadie H, Bellmunt J, Guo G, Cherniack AD, Hinoue T, Laird PW, Hoadley KA, Akbani R, et al. Comprehensive molecular characterization of muscle-invasive bladder cancer. Cell. 2017;171:540-56.

10. Sjodahl G, Eriksson P, Liedberg F, Hoglund M. Molecular classification of urothelial carcinoma: global mRNA classification versus tumour-cell phenotype classification. J Pathol. 2017;242:113-25.

11. Seiler R, Ashab H, Erho N, van Rhijn B, Winters B, Douglas J, Van Kessel KE, Fransen VDPE, Sommerlad M, Wang NQ, et al. Impact of molecular subtypes in muscle-invasive bladder cancer on predicting response and survival after neoadjuvant chemotherapy. Eur Urol. 2017;72:544-54.

12. van Rhijn BW, Vis AN, van der Kwast TH, Kirkels WJ, Radvanyi F, Ooms EC, Chopin DK, Boeve ER, Jobsis AC, Zwarthoff EC. Molecular grading of urothelial cell carcinoma with fibroblast growth factor receptor 3 and MIB-1 is superior to pathologic grade for the prediction of clinical outcome. J Clin Oncol. 2003;21:1912-21.

13. Neuzillet $Y$, Paoletti $X$, Ouerhani $S$, Mongiat-Artus $P$, Soliman $H$, de The $H$, Sibony M, Denoux Y, Molinie V, Herault A, et al. A meta-analysis of the relationship between FGFR3 and TP53 mutations in bladder cancer. Plos One. 2012;7:e48993.

14. Mazzola CR, Siddiqui KM, Billia M, Chin J. Dovitinib: rationale, preclinical and early clinical data in urothelial carcinoma of the bladder. Expert Opin Investig Drugs. 2014;23:1553-62.

15. Geelvink M, Babmorad A, Maurer A, Stohr R, Grimm T, Bach C, Knuechel R, Rose M, Gaisa NT. Diagnostic and prognostic implications of FGFR3(high)/ Ki67(high) papillary bladder cancers. Int J Mol Sci. 2018;19:2548-62.

16. Yang Z, Zhang R, Ge Y, Qin X, Kang X, Wang Y, Zhang X, Song C, Quan X, Wang $H$, et al. Somatic FGFR3 mutations distinguish a subgroup of muscleinvasive bladder cancers with response to neoadjuvant chemotherapy. Ebiomedicine. 2018;35:198-203.
17. Lerner SP. Targeted therapies for metastatic bladder cancer. J Urol. 2015;193 8-9.

18. Mayakonda A, Lin DC, Assenov Y, Plass C, Koeffler HP. Maftools: efficient and comprehensive analysis of somatic variants in cancer. Genome Res. 2018;28: $1747-56$.

19. Mroz EA, Rocco JW. MATH, a novel measure of intratumor genetic heterogeneity, is high in poor-outcome classes of head and neck squamous cell carcinoma. Oral Oncol. 2013;49:211-5.

20. Mroz EA, Tward AD, Hammon RJ, Ren Y, Rocco JW. Intra-tumor genetic heterogeneity and mortality in head and neck cancer: analysis of data from the Cancer Genome Atlas. Plos Med. 2015;12:e1001786.

21. Gao J, Aksoy BA, Dogrusoz U, Dresdner G, Gross B, Sumer SO, Sun Y,

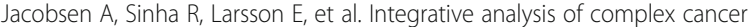
genomics and clinical profiles using the cBioPortal. Sci Signal. 2013;6:11.

22. Cerami E, Gao J, Dogrusoz U, Gross BE, Sumer SO, Aksoy BA, Jacobsen A, Byrne CJ, Heuer ML, Larsson E, et al. The cBio cancer genomics portal: an open platform for exploring multidimensional cancer genomics data. Cancer Discov. 2012;2:401-4.

23. Lane DP. Cancer. p53, guardian of the genome. Nature. 1992;358:15-6.

24. Kim PH, Cha EK, Sfakianos JP, Iyer G, Zabor EC, Scott SN, Ostrovnaya I, Ramirez R, Sun A, Shah R, et al. Genomic predictors of survival in patients with high-grade urothelial carcinoma of the bladder. Eur Urol. 2015;67:198201.

25. Cappellen D, De Oliveira C, Ricol D, de Medina S, Bourdin J, Sastre-Garau X, Chopin D, Thiery JP, Radvanyi F. Frequent activating mutations of FGFR3 in human bladder and cervix carcinomas. Nat Genet. 1999;23:18-20.

26. van Rhijn BW, Lurkin I, Radvanyi F, Kirkels WJ, van der Kwast TH, Zwarthoff EC. The fibroblast growth factor receptor 3 (FGFR3) mutation is a strong indicator of superficial bladder cancer with low recurrence rate. Cancer Res. 2001;61:1265-8.

27. Roberts SA, Lawrence MS, Klimczak LJ, Grimm SA, Fargo D, Stojanov P, Kiezun A, Kryukov GV, Carter SL, Saksena G, et al. An APOBEC cytidine deaminase mutagenesis pattern is widespread in human cancers. Nat Genet. 2013;45:970-6.

28. Han Y, Zheng Q, Tian Y, Ji Z, Ye H. Identification of a nine-gene panel as a prognostic indicator for recurrence with muscle-invasive bladder cancer. J Surg Oncol. 2019;119:1145-54.

29. Griffin JL. Devil in the detail: intratumour heterogeneity and personalised medicine for bladder cancer. Eur Urol. 2019;75:23-4.

30. Mazza P, Moran GW, Li G, Robins DJ, Matulay JT, Herr HW, Decastro GJ, McKiernan JM, Anderson CB. Conservative Management Following complete clinical response to neoadjuvant chemotherapy of muscle invasive bladder cancer: contemporary outcomes of a multi-institutional cohort study. J Urol. 2018;200:1005-13.

31. Turo R, Harnden P, Thygesen H, Fleischmann A, Thalmann GN, Seiler R, Cross WR, Knowles MA. FGFR3 expression in primary invasive bladder cancers and matched lymph node metastases. J Urol. 2015;193:325-30.

\section{Publisher's Note}

Springer Nature remains neutral with regard to jurisdictional claims in published maps and institutional affiliations.

Ready to submit your research? Choose BMC and benefit from:

- fast, convenient online submission

- thorough peer review by experienced researchers in your field

- rapid publication on acceptance

- support for research data, including large and complex data types

- gold Open Access which fosters wider collaboration and increased citations

- maximum visibility for your research: over $100 \mathrm{M}$ website views per year

At BMC, research is always in progress.

Learn more biomedcentral.com/submissions 